\title{
Direct Evidence for the Involvement of the Mesolimbic $\kappa$-Opioid System in the Morphine-Induced Rewarding Effect Under an Inflammatory Pain-Like State
}

\author{
Minoru Narita*,', Yayoi Kishimoto', Yuya Ise ${ }^{1,2}$, Yoshinori Yajima', Kaoru Misawa ${ }^{1,3}$ and Tsutomu Suzuki*,' \\ 'Department of Toxicology, Hoshi University School of Pharmacy and Pharmaceutical Sciences, Ebara, Shinagawa-ku, Tokyo, Japan; ${ }^{2}$ Department \\ of Pharmaceutical Service, Nippon Medical School Hospital, Sendagi Bunkyo-ku, Tokyo, Japan; ${ }^{3}$ Narcotic Drugs and Psychotropic Substances \\ Essential Drugs and Medicines Policy, World Health Organization, Geneva, Switzerland
}

\begin{abstract}
Recent clinical studies have demonstrated that when morphine is used to control pain in cancer patients, psychological dependence is not a major concern. The present study was undertaken to ascertain the modulation of psychological dependence on morphine under a chronic pain-like state in rats. The prototypical $\mu$-opioid receptor agonist morphine $(8 \mathrm{mg} / \mathrm{kg}$, i.p.) induced a dose-dependent place preference. In the present study, we found that an inflammatory pain-like state following formalin injection significantly suppressed the morphine-induced rewarding effect. This effect was almost reversed by s.c. pretreatment with the $\kappa$-opioid receptor antagonist norbinaltorphimine (nor-BNI, $5 \mathrm{mg} / \mathrm{kg}$ ). Furthermore, the morphine-induced increase in dopamine (DA) turnover in the limbic forebrain was significantly inhibited by treatment with formalin. This inhibition was also suppressed by pretreatment with nor-BNI. In addition, in vivo microdialysis studies clearly showed that the morphine-induced increase in the extracellular levels of DA and its metabolites, 3,4dihydroxyphenylacetic acid and homovanillic acid, in the nucleus accumbens (N.Acc.) was significantly decreased in rats that had been pretreated with formalin. This effect was in turn reversed by the microinjection of a specific dynorphin A antibody into the N.Acc. These findings suggest that the inflammatory pain-like state induced by formalin injection may have caused a sustained activation of the $\kappa$ opioidergic system within the N.Acc., resulting in suppression of the morphine-induced rewarding effect in rats. The present study provides further evidence of the clinical usefulness of morphine in patients suffering from severe pain.
\end{abstract}

Neuropsychopharmacology (2005) 30, I I I- | I 8, advance online publication, I 4 July 2004; doi: I 0. I038/sj.npp. I 300527

Keywords: opioid; inflammatory pain-like state; morphine dependence; nucleus accumbens; rat

\section{INTRODUCTION}

The World Health Organization publication Cancer Pain Relief (WHO, 1996) proposed a method for the relief of cancer pain based on a small number of relatively inexpensive drugs, including morphine. This approach is now used worldwide.

Recent clinical experience has shown that when morphine is used to control pain in cancer patients, psychological dependence is not a major concern (WHO, 1996). However, undue anxiety about psychological dependence on mor-

*Correspondence: M Narita, Department of Toxicology, Hoshi University School of Pharmacy and Pharmaceutical Sciences, 2-4-4I Ebara, Shinagawa-ku, Tokyo |42-850I, Japan, Tel/Fax: + 8135498 5628, E-mail: narita@hoshi.ac.jp and T Suzuki, Department of Toxicology, Hoshi University School of Pharmacy and Pharmaceutical Sciences, 2-4-4I Ebara, Shinagawa-ku, Tokyo I42-850 I, Japan, Tel/Fax: +81354985831,E-mail: suzuki@hoshi.ac.jp

Received 17 March 2003; revised 7 June 2004; accepted 9 June 2004 Online publication: 16 June 2004 at http://www.acnp.org/citations/ Npp06 |60404 | |4/default.pdf phine in cancer patients has caused physicians and patients to use inadequate doses of opioids. Nociceptive stimuli produced physiological changes in the levels of some proteins and neuropeptides in dorsal horn neurons (Dubner and Ruda, 1992; Narita et al, 2000). We reported previously that morphine failed to induce rewarding effects under a neuropathic pain-like state induced by sciatic nerve ligation in the rat and mouse (Ozaki et al, 2002, 2003). Furthermore, this pain-like state leads to a reduction in $\mu$-receptor function in the ventral tegmental area (VTA; Ozaki et al, 2002, 2003), which is the origin of the mesolimbic dopaminergic system and the major neural substrate of the rewarding effect produced by opioids (Koob, 1992; Nestler, 1996; Narita et al, 2001). These findings suggest that a state of pain could lead to physiological changes in neurotransmission at supraspinal levels, which could be responsible for the decrease in psychological dependence on opioids.

We also reported that morphine failed to induce rewarding effects under an inflammatory pain-like state produced by formalin or carrageenan (Suzuki et al, 1996, 
1999, 2001). However, there is, if any, direct evidence regarding possible changes at the supraspinal level after inflammation. Therefore, the aim of the present study was to investigate the modification of the rewarding effect induced by morphine under an inflammatory pain-like state produced by formalin injection.

\section{EXPERIMENTAL PROCEDURES}

The present study was conducted in accordance with the Guiding Principles for the Care and Use of Laboratory Animals, Hoshi University, as adopted by the Committee on Animal Research of Hoshi University, which is accredited by the Ministry of Education, Culture, Sports, Science, and Technology of Japan. Every effort was made to minimize the numbers and suffering of animals used in the following experiments.

\section{Animals}

Male Sprague-Dawley rats (200-300 g) were obtained from Tokyo Laboratory Animals Science, Co., Ltd (Tokyo, Japan). The rats were housed at a room temperature of $23 \pm 1^{\circ} \mathrm{C}$ with a $12 \mathrm{~h}$ light-dark cycle (light on $0830-2030 \mathrm{~h}$ ), and were allowed to adapt to this environment for 1 week before the experiments. Food and water were available ad libitum.

\section{Induction of Inflammation}

Formalin $(2.5 \%, 50 \mu \mathrm{l})$ or vehicle (saline, $100 \mu \mathrm{l})$ was injected into the plantar surface of the right hind paw. Rats treated with formalin were housed individually.

\section{Place Conditioning}

Place-conditioning studies were conducted using an apparatus consisting of a shuttle box (width $30 \mathrm{~cm} \times$ length $60 \mathrm{~cm} \times$ height $30 \mathrm{~cm}$ ) that was made of an acrylic resin board and divided into two colored compartments of equal size. One compartment was white with a textured floor and the other was black with a smooth floor. Conditioning sessions (two for morphine: two for saline) were started on the first day after formalin and vehicle injection into the rat paw, and conditioning was conducted once daily for 4 days (Suzuki et al, 1996, 1999, 2001). Immediately after i.p. injection of morphine $(2-8 \mathrm{mg} / \mathrm{kg})$, rats were placed in one compartment for $50 \mathrm{~min}$. On alternate days, rats were treated with saline and placed in the other compartment for $50 \mathrm{~min}$. The order of injection (morphine or saline) and compartment was counter-balanced (unbiased) across the subjects. The $\kappa$-opioid receptor antagonist nor-binaltorphimine (nor-BNI, $5 \mathrm{mg} / \mathrm{kg}$ ) was administered s.c. $6 \mathrm{~h}$ before i.p. treatment with morphine. On day 5 , tests of conditioning were performed as follows: the partition separating the two compartments was raised to $12 \mathrm{~cm}$ above the floor, a neutral platform was inserted along the seam separating the compartments, and rats that had not been treated with morphine or saline were placed on the platform. The time spent in each compartment during a $900 \mathrm{~s}$ session was then recorded automatically with an infrared beam sensor (KN80, Natsume Seisakusyo Co., Ltd, Tokyo, Japan). CPP scores represent the time spent in the drug (morphine)-paired place minus the time spent in the saline-paired place. All sessions were conducted under conditions of dim illumination (28lux lamp) and white masking noise. Each group consisted of $8-10$ rats.

\section{Quantification of Dopamine Turnover}

Using high-performance liquid chromatography with electrochemical detection (HPLC-ECD), the dopamine (DA), 3,4dihydroxyphenylacetic acid (DOPAC), homovanillic acid (HVA) levels were determined as previously described (Narita et al, 1993). Rats that had been pretreated with s.c. injection of nor-BNI $(5 \mathrm{mg} / \mathrm{kg})$ or saline were killed $30 \mathrm{~min}$ after s.c. injection of saline $(1 \mathrm{ml} / \mathrm{kg})$ or morphine $(5 \mathrm{mg} /$ $\mathrm{kg}$ ). The brain was removed quickly, and the limbic forebrain (containing nucleus accumbens (N.Acc.) and olfactory tubercles) and the striatum were dissected on an ice-cold metal plate. The tissues were homogenized in $500 \mu \mathrm{l}$ of $0.2 \mathrm{M}$ perchloric acid containing $100 \mu \mathrm{M}$ EDTA (2 Na) and $100 \mathrm{ng}$ isoproterenol as an internal standard. The homogenates were then centrifuged at $20000 \mathrm{~g}$ for $30 \mathrm{~min}$ at $4{ }^{\circ} \mathrm{C}$, and the supernatants were maintained at $\mathrm{pH} 3.0 \mathrm{using}$ $1 \mathrm{M}$ sodium acetate. Samples were analyzed by HPLC-ECD. The HPLC system consisted of a delivery system (EP-10, Eicom Co., Kyoto, Japan), an analytical column (Eicompac, MA-5ODS, Eicom Co.), and a guard column (Eicom Co.). DA and its metabolites were separated by a column with a mobile phase containing sodium acetate $(0.1 \mathrm{M})$, citric acid monohydrate $(0.1 \mathrm{M})$, sodium 1-octane sulfonate $(170 \mathrm{mg} / \mathrm{l})$, EDTA $(2 \mathrm{Na})(10 \mathrm{mg} / \mathrm{l})$, and $15 \%$ methanol. The mobile phase was delivered at a flow rate of $1.0 \mathrm{ml} / \mathrm{min}$. DA and its metabolites were identified according to the retention times of these standards, and the amounts were quantified by calculating the peak areas. The DA turnover, 'DA ratio', was calculated as (DOPAC + HVA)/DA.

\section{Surgery}

Stereotaxic surgery was performed under sodium pentobarbital $(50 \mathrm{mg} / \mathrm{kg}$, s.c.) anesthesia. Rats were placed in a stereotaxic apparatus and the skull was exposed. A small hole was then made using a dental drill. A guide cannula (AG-8, Eicom Co.) was implanted into the N.Acc. (AP: $+1.5 \mathrm{~mm}$ and L: $-1.5 \mathrm{~mm}$ from the bregma; $\mathrm{V}:-7.0 \mathrm{~mm}$ from the surface of the skull) according to the atlas of Paxinos and Watson (1998). The guide cannula was fixed to the skull with cranioplastic cement.

\section{Microinjection and In Vivo Microdialysis}

At 2-3 days after surgery, the animals were anesthetized with diethyl ether and injected with dynorphin A antibody $(1: 100)$ diluted in saline or saline alone in a volume of $2.0 \mu \mathrm{l} / \mathrm{rat}$ into the N.Acc. via a guide cannula using a Hamilton syringe at an infusion rate of $1.0 \mu \mathrm{l} / \mathrm{min}$. These infusions were delivered over $2 \mathrm{~min}$ and the rats were returned to their home cages after microinjection. At $1 \mathrm{~h}$ after microinjection, microdialysis probes (AI-8-2; $2 \mathrm{~mm}$ membrane length, Eicom Co.) were slowly inserted into the N.Acc. through the guide cannulas under anesthesia with diethyl ether, and rats were awakened and placed in the 
experimental cages (width $30 \mathrm{~cm} \times$ depth $30 \mathrm{~cm} \times$ height $30 \mathrm{~cm}$ ). The probes were perfused continuously at a flow rate of $2.0 \mu \mathrm{l} / \mathrm{min}$ with artificial cerebrospinal fluid (aCSF) containing $147.0 \mathrm{mM} \mathrm{NaCl}, 4.0 \mathrm{mM} \mathrm{KCl}$, and $2.3 \mathrm{mM} \mathrm{CaCl} 2$ (pH 5.4). Outflow fractions were taken every $20 \mathrm{~min}$. After three baseline fractions were collected, rats were treated with i.p. injection of morphine $(8 \mathrm{mg} / \mathrm{kg})$ or saline $(1 \mathrm{ml} / \mathrm{kg})$. For these experiments, dialysis samples were collected for $200 \mathrm{~min}$ after treatment with morphine or saline. Dialysis fractions were then analyzed using HPLC (Eicom Co.) with an ECD (Eicom Co.) system. The animals were killed by decapitation under sodium pentobarbital anesthesia at the end of the experiments. The brains were removed and the localization of the probes was confirmed by staining with Cresyl Violet.

\section{Quantification of Dopamine and Its Major Metabolites}

DA was separated by a column with a mobile phase containing sodium acetate $(3.95 \mathrm{~g} / \mathrm{l})$, citric acid monohydrate $(7.18 \mathrm{~g} / \mathrm{l})$, sodium 1 -octane sulfonate $(140 \mathrm{mg} / \mathrm{l})$, EDTA ( $2 \mathrm{Na} ; 5 \mathrm{mg} / \mathrm{l})$, and $17 \%$ methanol. The mobile phase was delivered at a flow rate of $0.21 \mathrm{ml} / \mathrm{min}$. DA, DOPAC, and HVA were identified according to the retention times of the standard, and amounts were quantified by calculating peak heights.

\section{Drugs}

The drugs used in the present study were formaldehyde solution (Wako Pure Chemical Ind., Ltd, Osaka, Japan), morphine hydrochloride (Sankyo Co., Tokyo, Japan), and dynorphin A antibody (1-17) (Phoenix Pharmaceuticals, Inc., Mountain View, CA, USA). The specificity of this antisera has been determined by radioimmunoassay or ELISA. Briefly, this antibody did not show any crossreactivities with endomorphin-1, endomorphin-2, Met-enkephalin, Leu-enkephalin-Lys, $\beta$-endorphin or $\beta$ neoendorphin (Tseng et al, 2000). Nor-BNI was synthesized by Dr Nagase (Toray Ind., Ltd, Kanagawa, Japan). All drugs were dissolved in sterile saline.

\section{Statistical Analysis}

Baseline microdialysis data were calculated as concentrations in the dialysates. Other microdialysis data were expressed as percentages of the corresponding baseline level. The data are expressed as the mean with SEM. The statistical analyses were performed using one-way and twoway analyses of variance with the Bonferroni/Dunn test.

\section{RESULTS}

The formalin-treated paws remained significantly swollen for 11 days $(\mathrm{F}(1,10)=511.54, p<0.001$, Figure 1a). Furthermore, mechanical hyperalgesia in the formalin-treated paw persisted for 9 days after formalin injection $(\mathrm{F}(1,16)=14.94$, $p<0.01$, Figure $1 \mathrm{~b}$ ). The time course of swelling paralleled that of the paw withdrawal threshold.

Under these conditions, we investigated whether the inflammatory pain-like state following formalin injection could affect the rewarding effect produced by morphine.
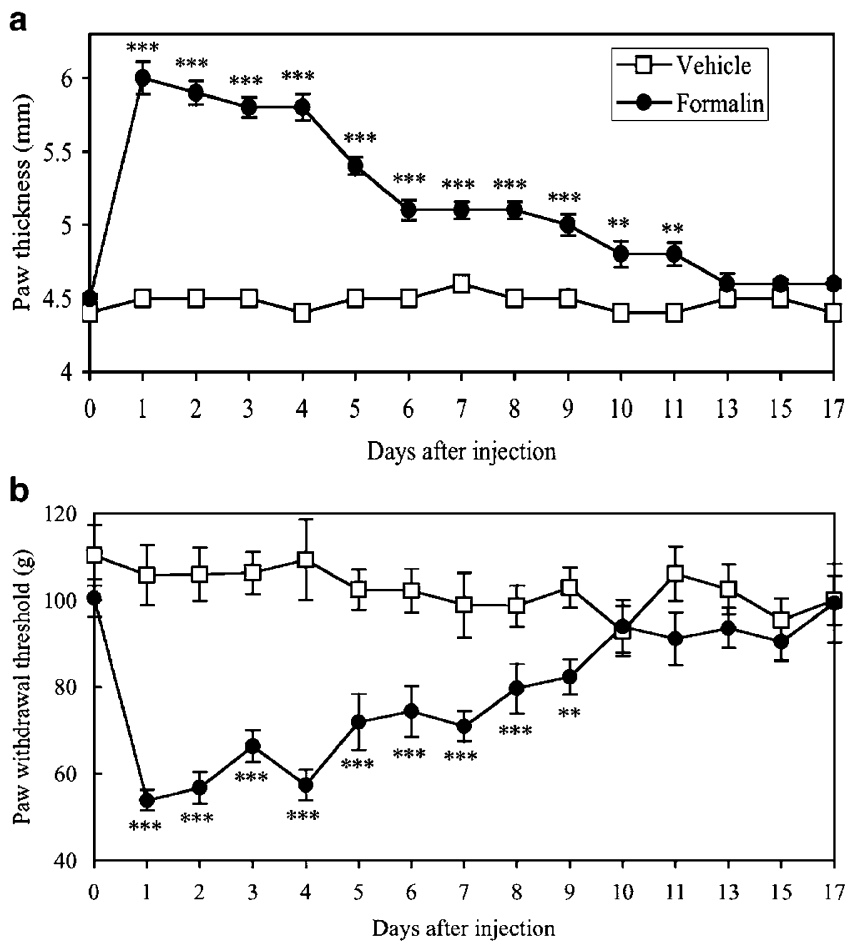

Figure I Time-course change in paw thickness (a) and withdrawal threshold (b) after the injection of formalin. Formalin-treated paws remained significantly swollen for II days. Furthermore, the pressure threshold in formalin-treated paws was markedly attenuated for 9 days. Each point represents the mean with SEM for 6 to 12 rats. $* * *<0.01$, **** $p<0.00$ I vs vehicle-treated group.

The i.p. injection of morphine produced a dose-related place preference in rats that had been treated with vehicle into the hind paw $(\mathrm{F}(3,30)=5.89, p<0.01$, Figure $2 \mathrm{a})$. This effect was significantly suppressed under the inflammatory pain-like state following formalin injection $(\mathrm{F}(1,15)=5.11$, $p<0.05$, Figure $2 \mathrm{a}$ ). In contrast, s.c. pretreatment with norBNI $(5 \mathrm{mg} / \mathrm{kg})$ dramatically reversed the suppression of the morphine $(8 \mathrm{mg} / \mathrm{kg})$-induced place preference in rats that had been treated with formalin $(\mathrm{F}(1,14)=4.89, p<0.05$, Figure $2 b$ ).

Since it is generally accepted that activation of the mesolimbic DAergic system is critically linked to the expression of the rewarding effect of morphine, we next investigated whether the suppression of the rewarding effect induced by morphine in rats treated with formalin could result from the changes in DA turnover in the limbic forebrain region including the N.Acc. The contents of DA and its metabolites in the rat limbic forebrain and striatum are shown in Table 1. None of the vehicle- or formalintreated rat brain tissues exhibited a significant difference in the contents of DA and its metabolites. The DA turnover in the limbic forebrain and striatum was significantly increased by i.p. injection of morphine (limbic forebrain; $\mathrm{F}(1,10)=132.77, \quad p<0.001, \quad$ striatum; $\mathrm{F}(1,10)=158.45$, $p<0.001$, Figure 3a,b). The morphine-induced increase in DA turnover in the limbic forebrain region, but not the striatum, was significantly suppressed in formalin-treated rats (limbic forebrain; $\mathrm{F}(1,10)=5.50, p<0.05$, striatum; $\mathrm{F}(1,10)=0.09, \quad \mathrm{NS}$, Figure $3 \mathrm{a}, \mathrm{b})$. Interestingly, this 


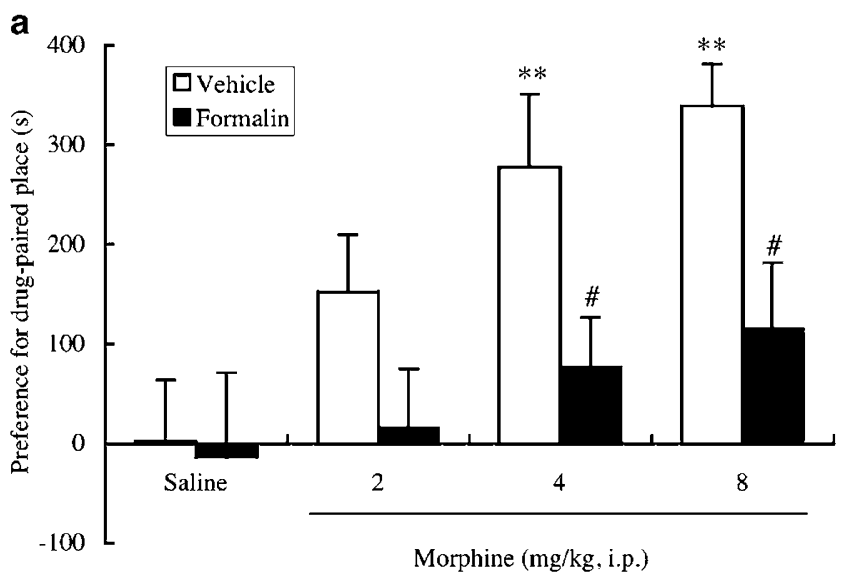

Table I Levels of Dopamine (DA) and Its Major Metabolites (DOPAC and HVA) in the Limbic Forebrain and Striatum of Rats Treated with Morphine or nor-BNI (5 mg/kg) Following the Injection of Vehicle or Formalin

\begin{tabular}{lccc}
\hline & \multicolumn{3}{c}{ Contents (ng/g of wet tissue) } \\
\cline { 2 - 4 } & DA & DOPAC & HVA \\
\hline $\begin{array}{c}\text { Limbicfore brain } \\
\text { Saline (i.p.) }\end{array}$ & & & \\
Vehicle+Saline & $9192.5 \pm 771.6$ & $1030.3 \pm 73.4$ & $694.0 \pm 99.3$ \\
Vehicle+nor-BNI & $6561.3 \pm 412.9$ & $846.9 \pm 50.1$ & $533.3 \pm 43.9$ \\
Formalin+Saline & $8683.7 \pm 52.1$ & $998.2 \pm 29.2$ & $678.7 \pm 31.3$ \\
Formalin+nor-BNI & $5763.9 \pm 367.9$ & $635.9 \pm 53.0$ & $445.5 \pm 27.2$ \\
& & & \\
Morphine (8 mg/kg i.p.) & & & \\
Vehicle+Saline & $9649.9 \pm 419.8$ & $2011.0 \pm 157.0$ & $1486.7 \pm 82.3$ \\
Vehicle+nor-BNI & $10791.7 \pm 370.0$ & $2068.1 \pm 99.4$ & $1566.9 \pm 77.1$ \\
Formalin+Saline & $8635.1 \pm 684.6$ & $1358.8 \pm 54.6$ & $1030.3 \pm 38.6$ \\
Formalin+nor-BNI & $10591.2 \pm 283.2$ & $2037.3 \pm 89.3$ & $1484.9 \pm 48.2$
\end{tabular}

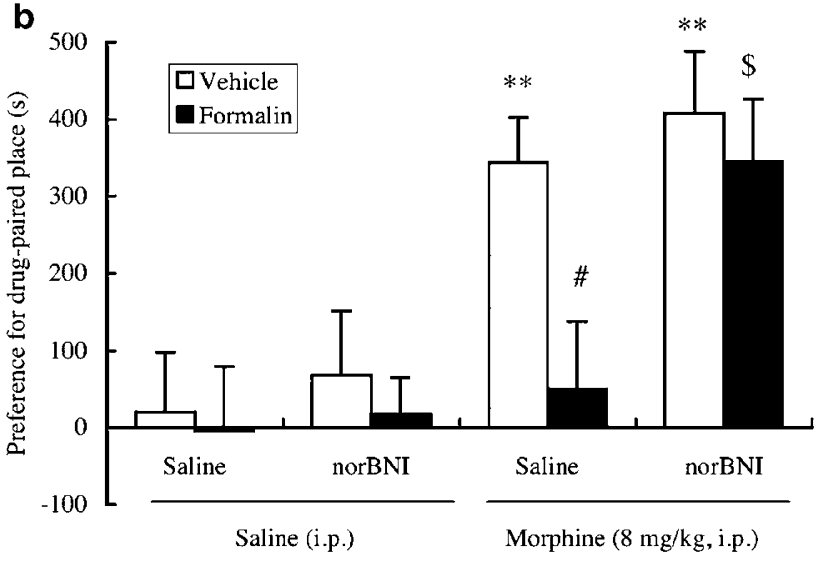

Figure 2 Effect of pretreatment with nor-BNI on the suppression of the place preference induced by morphine $(2-8 \mathrm{mg} / \mathrm{kg}$, i.p.) under an inflammatory pain-like state. (a) The morphine-induced rewarding effect was suppressed in rats treated with formalin. (b) The suppression of the morphine-induced rewarding effect by formalin injection was reversed by s.c. pretreatment with nor-BNI $(5 \mathrm{mg} / \mathrm{kg})$. The ordinate represents the preference for the drug-paired place. Each column represents the mean with SEM for 8 to 10 rats. $* * *<0.01$ vs Vehicle/Saline/Saline group, ${ }^{\#} p<0.05$ vs Vehicle/Saline/Morphine group, ${ }^{\$} p<0.05$ vs Formalin/Saline/ Morphine group.

suppression of morphine-induced DA turnover in formalintreated rats was significantly reversed by pretreatment with $5 \mathrm{mg} / \mathrm{kg}$ of nor-BNI $(\mathrm{F}(1,10)=14.98, p<0.01$, Figure $3 \mathrm{a})$.

We next demonstrated using in vivo microdialysis studies whether the $\kappa$-opioidergic system was responsible for suppression of the morphine-induced activation of mesolimbic DA neurons under an inflammatory pain-like state. Figure 4 shows the placement of microdialysis probes within the N.Acc. Regions that contained probes were localized in the N.Acc. Only data from rats in which probes had been accurately inserted in the N.Acc. were used for subsequent statistical analysis. There were no differences in levels of DA, DOPAC, and HVA among the groups (Table 2). The extracellular level of DA in the N.Acc. was markedly increased by i.p. injection of morphine $(8 \mathrm{mg} / \mathrm{kg})$ in the vehicle-treated control group (Vehicle/Saline/Saline group vs Vehicle/Saline/Morphine group, $\mathrm{F}(1,84)=15.61$, $p<0.001$, Figure 5), whereas it was significantly decreased by pretreatment with formalin in the hind paw (Vehicle/ Saline/Morphine group vs Formalin/Saline/Morphine group, $\mathrm{F}(1,96)=25.53, p<0.001$, Figure 5). Microinjection of the specific antibody to dynorphin A into the N.Acc. did not affect the morphine-induced increase in the extracellular level of DA in the vehicle-treated control group. Under these conditions, the suppression of the morphineinduced increase in the extracellular level of DA in the N.Acc. produced by intraplantar injection of formalin was almost reversed by the microinjection of dynorphin A antibody into the N.Acc. (Formalin/Saline/Morphine group vs Formalin/Dynorphin A antibody/Morphine group, $\mathrm{F}(1,96)=19.61, p<0.01$, Figure 5).

The i.p. injection of morphine also produced a significant increase in the major DA metabolites DOPAC (Vehicle/ Saline/Saline group $v s$ Vehicle/Saline/Morphine group, $\mathrm{F}(1,84)=41.69, p<0.001$, Figure 6a) and HVA (Vehicle/ Saline/Saline group vs Vehicle/Saline/Morphine group, $\mathrm{F}(1,94)=93.79, p<0.001$, Figure $6 \mathrm{~b})$ in the vehicle-treated group. In contrast, these effects were significantly attenuated in rats that had been treated with formalin (DOPAC; Vehicle/Saline/Morphine group vs Formalin/Saline/ 
a

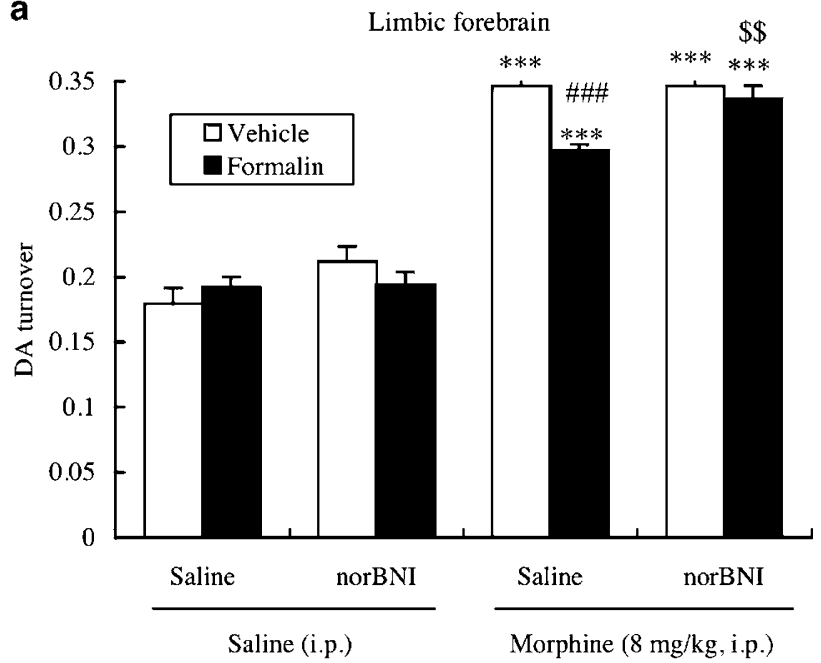

b

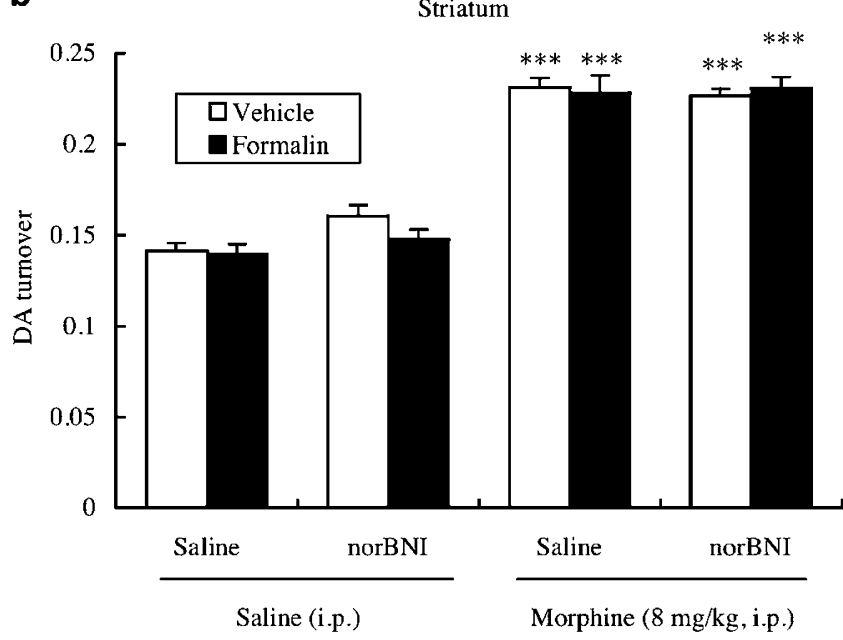

Figure 3 Effect of pretreatment with nor-BNI $(5 \mathrm{mg} / \mathrm{kg}$, s.c.) on the suppression of DA turnover induced by morphine $(8 \mathrm{mg} / \mathrm{kg}$, i.p.) under an inflammatory pain-like state. The morphine-induced increase in DA turnover in the limbic forebrain (a), but not the striatum (b), was suppressed in rats that had been treated with formalin. The suppression of morphine-induced DA turnover by formalin injection was reversed by s.c. pretreatment with nor-BNI. Each column represents the mean with SEM of 6-8 rats. The DA turnover (DA ratio) was calculated as (DOPAC + HVA)/ DA. ${ }^{* * *} * x<0.00$ I vs Vehicle/Saline or nor-BNI/Saline group, $\# \# \#<0.00$ I Vehicle/Saline/Morphine group vs Formalin/Saline/Morphine group, $\$ \$ p<0.0$ I Formalin/Saline/Morphine group vs Formalin/nor-BNI/Morphine group.

Morphine group, $\mathrm{F}(1,84)=41.69, p<0.001$, Figure 6a; HVA; Vehicle/Saline/Morphine group vs Formalin/Saline/Morphine group, $\mathrm{F}(1,96)=54.20, p<0.001$, Figure $6 \mathrm{~b})$. Furthermore, the suppression of the morphine-induced increase in the extracellular level of DA metabolites in the N.Acc. in rats that had been treated with formalin was almost reversed by the intra-N.Acc. injection of dynorphin A antibody (DOPAC; Formalin/Saline/Morphine group vs Formalin/ Dynorphin A antibody/Morphine group, $\mathrm{F}(1,84)=8.69$, $p<0.05$, Figure 6a; HVA; Formalin/Saline/Morphine group $v s$ Formalin/Dynorphin A antibody/Morphine group, $\mathrm{F}(1,108)=25.53, p<0.001$, Figure $6 \mathrm{~b})$.

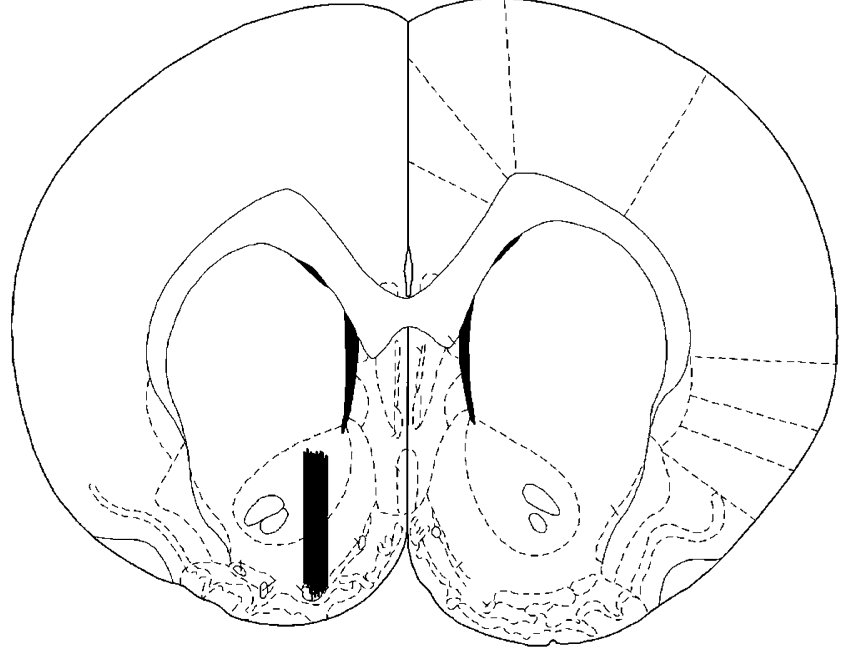

Bregma $1.6 \mathrm{~mm}$

Figure 4 Localization of microdialysis probes in the N.Acc. in rats. Stippled lines represent regions in the rat brain that contained probes. The schematic brain sections are from the atlas of Paxinos and Watson (1998).

Table 2 Basal Dialysate Levels of Dopamine (DA) and Its Major Metabolites (DOPAC and HVA) in the N.Acc. of Vehicle- or Formalin-Treated Rats

\begin{tabular}{lccc}
\hline Group & DA (nM) & DOPAC (nM) & HVA (nM) \\
\hline Vehicle/Saline/Saline & $1.14 \pm 0.22$ & $258.33 \pm 51.96$ & $230.09 \pm 34.73$ \\
Vehicle/Saline/Morphine & $1.13 \pm 0.11$ & $289.22 \pm 39.77$ & $210.04 \pm 26.16$ \\
Formalin/Saline/Saline & $1.42 \pm 0.39$ & $236.08 \pm 70.58$ & $191.97 \pm 51.56$ \\
Formalin/Saline/Morphine & $1.22 \pm 0.28$ & $297.74 \pm 57.86$ & $196.50 \pm 30.62$ \\
Vehicle/Anti-Dyn A/Morphine & $1.07 \pm 0.15$ & $278.84 \pm 49.56$ & $248.03 \pm 33.42$ \\
Formalin/Anti-Dyn A/Morphine & $1.14 \pm 0.34$ & $310.07 \pm 71.68$ & $237.95 \pm 47.85$
\end{tabular}

Each value represents the mean with SEM of 6 to 8 rats.

\section{DISCUSSION}

Many studies have pointed to the mesolimbic DAergic system, projecting from the VTA of the midbrain to the N.Acc., as the critical substrate of the rewarding effect of morphine (Koob, 1992; Nestler, 1996; Narita et al, 2001). $\mu$-Opioid receptor agonists have been shown to increase DAergic signals in the N.Acc. via the activation of DA cells in the VTA, an area that possesses high densities of $\mu$-opioid receptors (Garzon and Pickel, 2001). We reported previously that the enhancement of DA turnover in the mesolimbic forebrain area containing the N.Acc. induced by morphine was associated with the expression of a rewarding effect by morphine (Funada et al, 1993; Narita et al, 2001). In the present study, we found that the injection of formalin into the plantar surface of the hind paw suppressed both the rewarding effect and activation of the mesolimbic DA system induced by morphine in rats. These results suggest that the mesolimbic DA system may not be facilitated by $\mu$-opioid receptor agonists under an inflammatory pain-like state. 


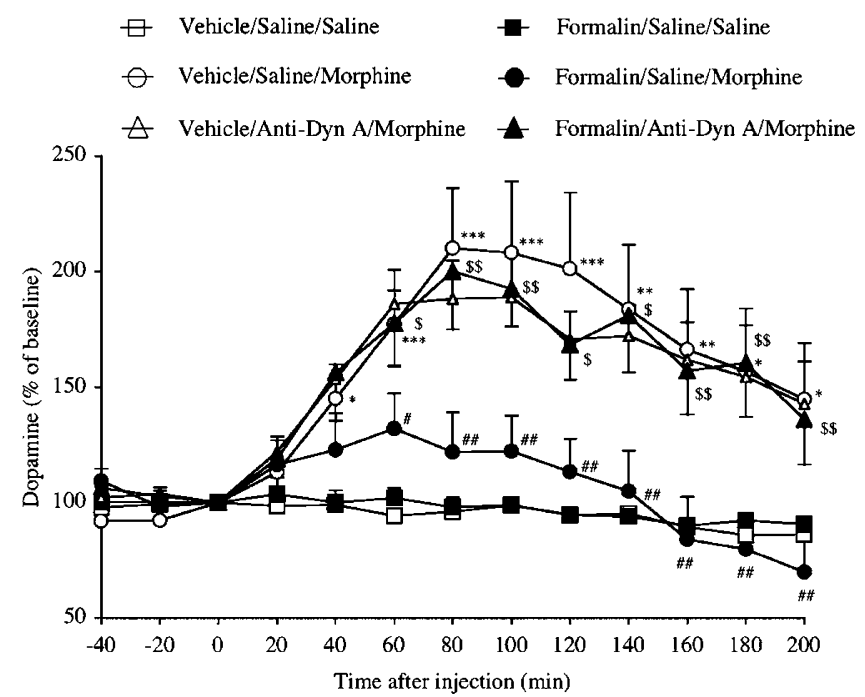

Figure 5 Effect of pretreatment with an antibody specific to dynorphin A (Anti-Dyn A) into the N.Acc. on the extracellular level of dopamine in the N.Acc. induced by morphine ( $8 \mathrm{mg} / \mathrm{kg}$, i.p.) under an inflammatory painlike state. The morphine-induced increase in dopamine release in the N.Acc. was suppressed in rats that had been treated with formalin. The suppression of the morphine-induced dopamine release by formalin injection was reversed by the intra-N.Acc. injection of Anti-Dyn A. Data are expressed as a percentage of the corresponding baseline levels with SEM of 4 to 5 rats. $* p<0.05$, $* * p<0.0$ I, **** $p<0.00$ I Vehicle/Saline/Saline group vs Vehicle/Saline/Morphine group. ${ }^{\#} p<0.05,{ }^{\# \#} p<0.0$ I Vehicle/ Saline/Morphine group vs Formalin/Saline/Morphine group. $\$ p<0.05$, $\$ \$>0.01$ Formalin/Saline/Morphine group vs Formalin/Anti-Dyn A/ Morphine group.

In a previous study, we observed that intra-VTA microinjection of the selective $\mu$-opioid receptor agonist $\left[\mathrm{D}-\mathrm{Ala}^{2}, \mathrm{NMPhe}^{4}, \mathrm{Gly}(\mathrm{ol})^{5}\right]$ enkephalin (DAMGO) produced a significant decrease in the extracellular $\gamma$-amino butyric acid (GABA) level in the VTA, indicating that disinhibition may be the key role of $\mu$-opioid receptor-mediated function in the VTA. This contention can be supported by the finding that morphine and DAMGO each inhibits the firing frequency of non-DA cells in the VTA (Johnson and North, 1992; Bonci and Williams, 1997). These findings strongly suggest that the activation of $\mu$-opioid receptor in the VTA may facilitate the mesolimbic DA system through the inhibition of GABAergic neurotransmission in the VTA, resulting in the induction of a rewarding effect induced by $\mu$-opioid receptor agonists. Considering this background, we first hypothesized that either GABAergic or $\mu$-opioidergic systems in the VTA could be altered by formalin injection. However, this is not a major effect, since the baseline levels of DA and its metabolites in the N.Acc. were not affected at all under an inflammatory pain-like state. These findings support the idea that other modulatory systems linked to the mesolimbic DA system might be altered under an inflammatory pain-like state.

Several lines of evidence indicate that the $\kappa$-opioidergic system negatively modulates the DA-related actions mediated by $\mu$-opioid receptors (Funada et al, 1993; Narita et al, 1993, 2001). We found previously that pretreatment with the $\kappa$-opioid receptor agonist $\mathrm{U} 50,488 \mathrm{H}$ at a dose that alone did not produce place aversion suppressed both the place preference and the elevation of DA metabolites in the rat limbic forebrain produced by morphine (Funada et al,
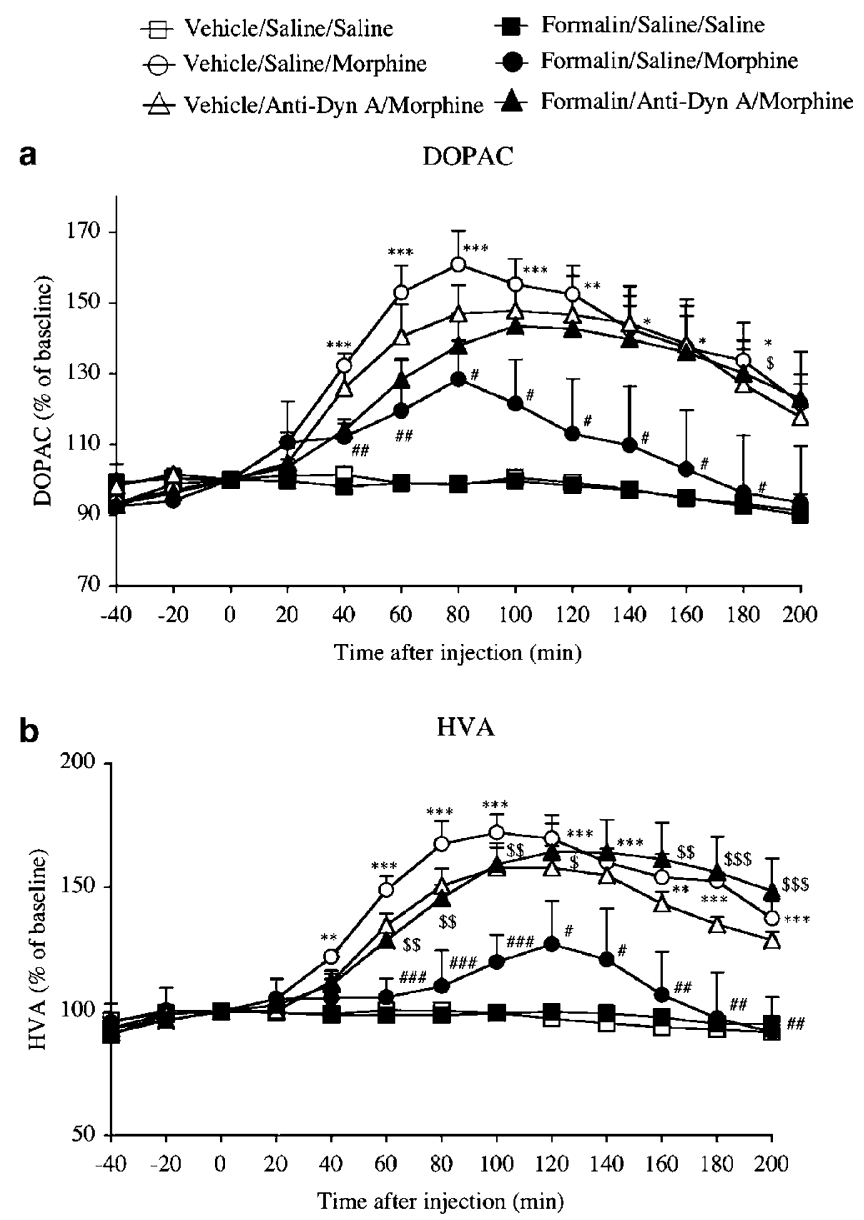

Figure 6 Effect of pretreatment with an antibody specific to dynorphin A (Anti-Dyn A) into the N.Acc. on the extracellular levels of DOPAC (a) and HVA (b) in the N.Acc. induced by morphine $(8 \mathrm{mg} / \mathrm{kg}$, i.p.) under an inflammatory pain-like state. The morphine-induced increase in DOPAC and HVA in the N.Acc. was suppressed in rats that had been treated with formalin. The suppression of the morphine-induced increase in the extracellular levels of DOPAC and HVA was reversed by the intraN.Acc. injection of Anti-Dyn A in rats that had been treated with formalin. Data are expressed as a percentage of the corresponding baseline levels with SEM of 4 to 5 rats. ${ }^{*} p<0.05$, *** $p<0.0$ I, **** $p<0.00$ I Vehicle/Saline/ Saline group vs Vehicle/Saline/Morphine group. ${ }^{\#} p<0.05,{ }^{\# \#} p<0.0$ I,

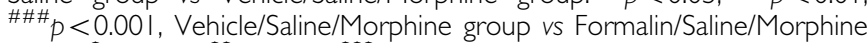
group. ${ }^{\$} p<0.05,{ }^{\$ \$} p<0.01,{ }^{\$ \$ \$} p<0.001$, Formalin/Saline/Morphine group vs Formalin/Anti-Dyn A/Morphine group.

1993; Narita et al, 2001). In addition, the increase in the extracellular level of DA produced by morphine was attenuated by the microinjection of $\kappa$-opioid receptor agonist into the N.Acc., but not into the VTA (Spanagel et al, 1992), implying that the $\kappa$-opioidergic system within the N.Acc. may play an important role in the negative modulation of the mesolimbic DA-dependent motivational effect produced by $\mu$-opioid receptor agonists.

Previous studies have demonstrated that the levels of dynorphin and pre-prodynorphin mRNA in some brain regions are increased under inflammatory pain-like states induced by the injection of carrageenan and complete Freund's adjuvant (Millan et al, 1987; Dubner and Ruda, 1992). These findings suggest that a state of chronic pain could lead to physiological changes in $\kappa$-opioidergic neurotransmission in the brain. In the present study, we 
found that the elevation of DA turnover in the limbic forebrain induced by morphine was significantly suppressed by formalin injection and this effect was reversed by the selective $\kappa$-opioid receptor antagonist nor-BNI. In addition, the suppression of the morphine-induced increase in the extracellular levels of DA, DOPAC, and HVA in the N.Acc. following formalin injection was also reversed by the microinjection of an antibody specific to dynorphin A into the N.Acc. of rats that had been treated with formalin. Dynorphin A antibody failed to affect the basal level of DA in the N.Acc. following formalin injection, implying that this antibody has a specific effect on the morphine-induced increase in the extracellular levels of DA in the N.Acc. The injection of formalin did not directly affect the basal levels of DA, DOPAC, and HVA. These results support the fascinating possibility that the mild or moderate increase in the release of dynorphin A from $\kappa$-opioidergic terminals within the N.Acc. under an inflammatory pain-like state may not affect the basal state of the mesolimbic DA system. However, it may inhibit the overshooting of the mesolimbic DA system facilitated by morphine, resulting in suppression of the morphine-induced rewarding effect (Figure 7).
Endogenous $\mu$ - and $\kappa$-opioidergic systems can be physiologically balanced under a normal state. After morphine treatment, $\mu$-opioidergic systems are superfluously excited, which may in turn upset the balance of the endogenous $\mu$ - and $\kappa$-opioidergic systems. This disruption may lead to the development of psychological dependence on morphine under a normal state. On the other hand, inflammatory pain may facilitate the endogenous $\kappa$-opioidergic system within the N.Acc., leading to negative modulation of the mesolimbic DA system. Under this condition, the biased balance of endogenous $\mu$ - and $\kappa$ opioidergic systems due to inflammation may actually be improved by morphine treatment. Therefore, psychological dependence on morphine may not be developed under an inflammatory pain-like state (Figure 8).

In conclusion, we demonstrated here that both the rewarding effect and facilitation of the mesolimbic DA system produced by morphine were significantly suppressed in rats that had been treated with formalin. These effects were reversed by blockade of the endogenous $\kappa$ opioidergic system in the N.Acc. These findings raise the possibility that an inflammatory pain-like state may cause a

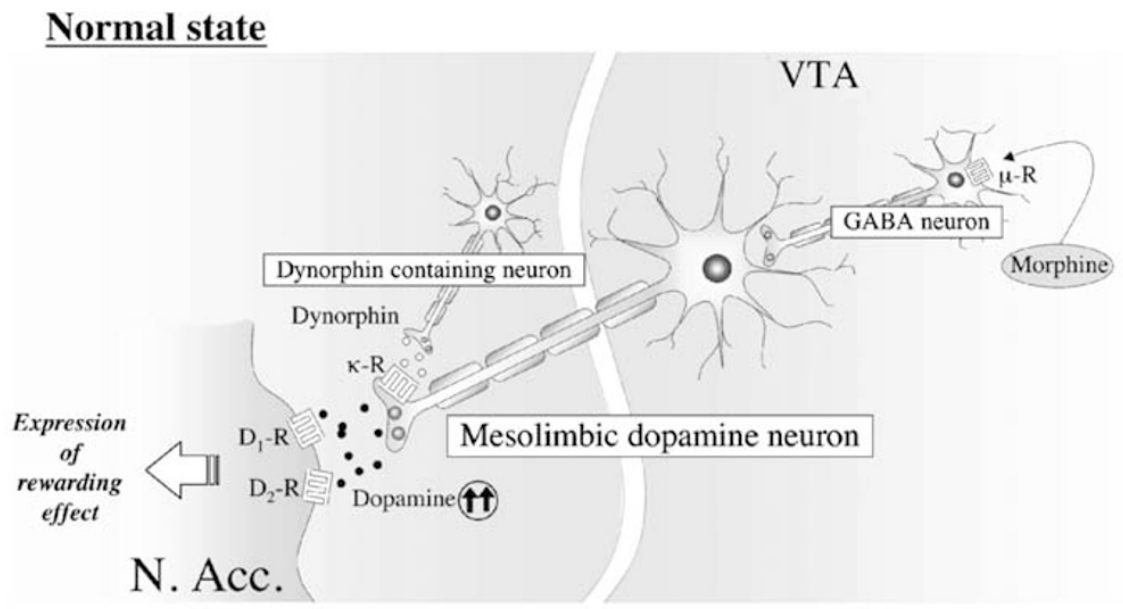

\section{Inflammatory pain-like state}

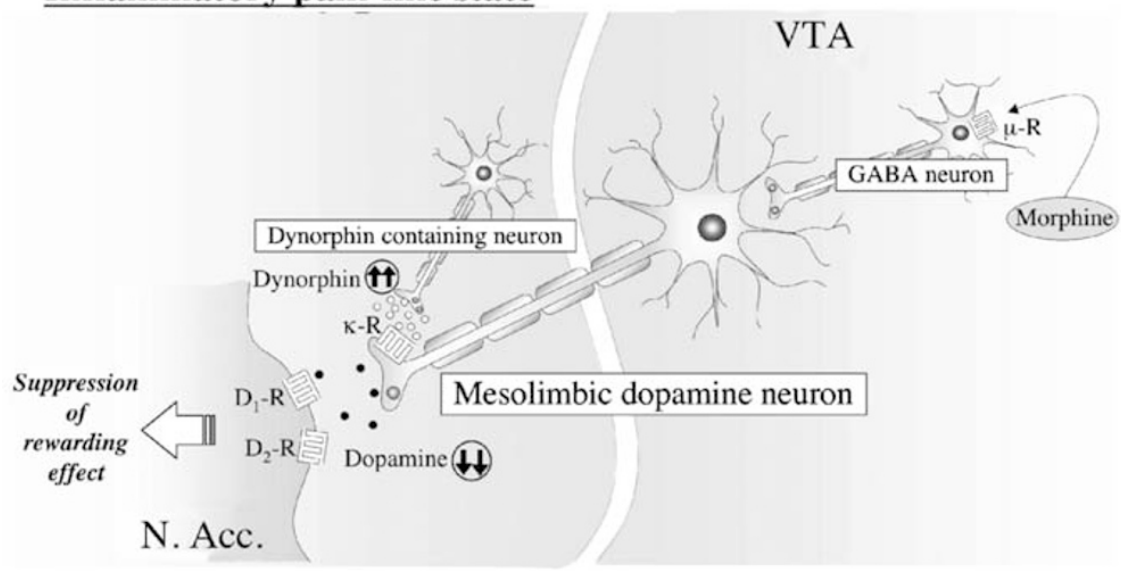

Figure 7 A schematic drawing of the suppression of the rewarding effect produced by morphine under an inflammatory pain-like state. In a normal state, morphine produces an increase in dopamine release in the N.Acc. through disinhibition of the GABAergic system in the VTA, resulting in the expression of a rewarding effect. In contrast, the morphine-induced rewarding effect is suppressed under an inflammatory pain-like state, due to the inhibition of dopamine release at dopaminergic terminals through facilitation of the endogenous $\kappa$-opioidergic system within the N.Acc. 
a

Normal state

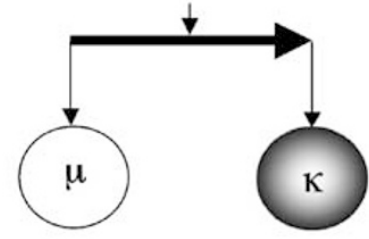

b

Normal state with morphine

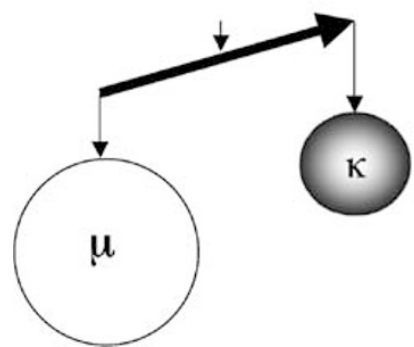

Figure 8 A schematic drawing of the presumed physiological balance between the endogenous $\mu$ - and $\kappa$-opioidergic systems within the mesolimbic dopaminergic system under normal and inflammatory painlike states. (a) Endogenous $\mu$ - and $\kappa$-opioidergic systems may be balanced under a normal state. (b) Since $\mu$-opioidergic systems are superfluously facilitated by morphine, psychological dependence on morphine develops. (c) On the other hand, inflammatory pain may cause a marked activation of the endogenous $\kappa$-opioidergic system. (d) Several lines of evidence have demonstrated that activation of the $\kappa$-opioidergic system suppresses the morphine-induced rewarding effect in rodents. Thus, we propose that psychological dependence on morphine may not develop under a state of inflammatory pain due to the activation of endogenous $\kappa$-opioidergic systems. Therefore, adequate treatment of patients with morphine is highly recommended for the relief of severe chronic pain.

sustained facilitation of the $\kappa$-opioidergic system within the N.Acc., resulting in suppression of the morphine-induced rewarding effect in rats.

\section{ACKNOWLEDGEMENTS}

This work was supported in part by grants from the Ministry of Health, Labor and Welfare, and the Ministry of Education, Culture, Sports, Science and Technology of Japan.

\section{REFERENCES}

Bonci A, Williams J (1997). Increased probability of GABA release during withdrawal from morphine. J Neurosci 17: 796-803.

Dubner R, Ruda MA (1992). Activity-dependent neuronal plasticity following tissue injury and inflammation. Trends Neurosci 15: 96-103.
Funada M, Suzuki T, Narita M, Misawa M, Nagase H (1993). Blockade of morphine reward through the activation of $\kappa$-opioid receptors in mice. Neuropharmacology 32: 1315-1323.

Garzon M, Pickel VM (2001). Plasmalemmal $\mu$-opioid receptor distribution mainly in nondopaminergic neurons in the rat ventral tegmental area. Synapse 41: 311-328.

Johnson SW, North RA (1992). Opioids excite dopamine neurons by hyperpolarization of local interneurons. J Neurosci 12: 483-488.

Koob GF (1992). Drugs of abuse: anatomy, pharmacology and function of reward pathways. Trends Pharmacol Sci 13: 177-184.

Millan MJ, Czlonkowski A, Pilcher CW, Almeida OF, Millan MH, Colpaert FC et al (1987). A model of chronic pain in the rat: functional correlates of alterations in the activity of opioid systems. J Neurosci 7: 77-87.

Narita M, Funada M, Suzuki T (2001). Regulations of opioid dependence by opioid receptor types. Pharmacol Ther 89: 1-15.

Narita M, Suzuki T, Funada M, Misawa M, Nagase H (1993). Blockade of the morphine-induced increase in turnover of dopamine on the mesolimbic dopaminergic system by $\kappa$-opioid receptor activation in mice. Life Sci 52: 397-404.

Narita M, Yajima Y, Aoki T, Ozaki S, Mizoguchi H, Tseng LF et al (2000). Up-regulation of the TrkB receptor in mice injured by the partial ligation of the sciatic nerve. Eur J Pharmacol 401: 187-190.

Nestler EJ (1996). Under Siege: the brain on opiates. Neuron 16: 897-900.

Ozaki S, Narita M, Narita M, Iino M, Miyoshi K, Suzuki T (2003). Suppression of the morphine-induced rewarding effect and Gprotein activation in the lower midbrain following nerve injury in the mouse: involvement of G-protein-coupled receptor kinase 2. Neuroscience 116: 89-97.

Ozaki S, Narita M, Narita M, Iino M, Sugita J, Matsumura Y, Suzuki T (2002). Suppression of the morphine-induced rewarding effect in the rat with neuropathic pain: implication of the reduction in $\mu$-opioid receptor functions in the ventral tegmental area. J Neurochem 82: 1192-1198.

Paxinos G, Watson C (1998). The Rat Brain in Stereotaxic Coordinates. Academic Press: New York.

Spanagel R, Herz A, Shippenberg T (1992). Opposing tonically active endogenous opioid systems modulate the mesolimbic dopaminergic pathway. Proc Natl Acad Sci USA 89: 2046-2050.

Suzuki T, Kishimoto Y, Misawa M (1996). Formalin- and carrageenan-induced inflammation attenuates place preferences produced by morphine, methamphetamine and cocaine. Life Sci 59: $1667-1674$.

Suzuki T, Kishimoto Y, Misawa M, Nagase H, Takeda F (1999). Role of the $\kappa$-opioid system in the attenuation of the morphineinduced place preference under chronic pain. Life Sci 64: PL1-PL7.

Suzuki T, Kishimoto Y, Ozaki S, Narita M (2001). Mechanism of opioid dependence and interaction between opioid receptors. Eur J Pain 5(Suppl A): 63-65.

Tseng LF, Narita M, Suganuma C, Mizoguchi H, Ohsawa M, Nagase $\mathrm{H}$ et al (2000). Differential antinociceptive effects of endomorphin-1 and endomorphin-2 in the mouse. J Pharmacol Exp Ther 292: 576-583.

World Health Organization (1996). Cancer Pain Relief. World Health Organization: Geneva. pp 24-37. 